\title{
Kaon photoproduction: Background contributions, form factors, and missing resonances
}

\author{
Stijn Janssen, ${ }^{*}$ Jan Ryckebusch, Dimitri Debruyne, and Tim Van Cauteren \\ Department of Subatomic and Radiation Physics, Ghent University, Proeftuinstraat 86, B-9000 Gent, Belgium
}

(Received 12 July 2001; published 30 November 2001)

\begin{abstract}
The photoproduction $p\left(\gamma, K^{+}\right) \Lambda$ process is studied within a field-theoretic approach. It is shown that the background contributions constitute an important part of the reaction dynamics. We compare predictions obtained with three plausible techniques for dealing with these background contributions. It appears that the extracted resonance parameters drastically depend on the applied technique. We investigate the implications of the corrections to the functional form of the hadronic form factor in the contact term, recently suggested by Davidson and Workman [Phys. Rev. C 63, 025210 (2001)]. The role of background contributions and hadronic form factors for the identification of the quantum numbers of "missing" resonances is discussed.
\end{abstract}

DOI: 10.1103/PhysRevC.65.015201

PACS number(s): 13.60.Le, 13.30.Eg, 14.20.Gk, 14.20.Jn

\section{INTRODUCTION}

Gaining deeper insight into the structure of the nucleon is one of the ultimate goals of current research in mediumenergy physics. One of the crucial topics is understanding the excited states of the nucleon, in brief denoted as $N^{*}$. Most of the available information concerning nucleon resonances is based on the knowledge extracted from electromagnetically induced pion production and pion-induced reactions. Despite the fact that invaluable information regarding $N^{*}$ 's is obtained in the pion sector, for a long time it has been realized that alternate meson production reactions could provide additional information on the excitation spectrum of the nucleon. In particular, the involvement of a strange $q \bar{q}$-quark pair in the reaction opens an additional degree of freedom, and it is believed that some of the (unobserved) resonances have specific strong coupling into these "strange channels" [1].

At present, high-duty electron and photon facilities like CEBAF, ELSA, MAMI, Spring-8, GRAAL, Bates, and LEGS provide data for electroproduction and photoproduction of mesons with unprecedented accuracy. One of the major challenges for the field is extracting from the data reliable information about resonances, like photocoupling helicity amplitudes, and strong decay widths, in as modelindependent a fashion as possible. In principle, a complete coupled-channel analysis could handle the challenging problem of extracting the relevant physics from the mesoninduced and meson production reaction data. Over the last couple of years, there has been quite some progress in this field, and several frameworks to perform a combined analysis of the photon and meson induced reactions have been developed [2-5]. Apart from a unified description of a wide variety of reactions, a coupled-channel approach can incorporate the effect of final-state interactions into a description of the dynamics. A recent study [5] reported that these finalstate interaction effects on the computed $p\left(\gamma, K^{+}\right) \Lambda$ cross sections are of the order of $20 \%$. Apart from the uncertainties inherent to coupled-channel approaches, such as unknown

*Email address: stijn.janssen@rug.ac.be phase shifts and off-shell rescattering ambiguities, these results indicate that a large part of the $p\left(\gamma, K^{+}\right) \Lambda$ reaction dynamics is dominated by first-order, so-called, "tree level" diagrams. In this work, we show that in a tree level description of the $p\left(\gamma, K^{+}\right) \Lambda$ process, a reliable extraction of resonance parameters is still far from evident and subject to uncertainties. We believe that, in addition to directing efforts toward dealing with the coupled-channel final-state interaction effects, a proper treatment and understanding of the first-order tree level terms is absolutely necessary. The primary goal of this work is to quantify the model dependency of the extracted resonance parameters due to the uncertainties stemming from the background contributions and the introduction of hadronic form factors.

The outline of this paper is as follows. In Sec. II, we briefly discuss the field-theoretic formalism to describe the reaction dynamics of the $p\left(\gamma, K^{+}\right) \Lambda$ process. In Sec. III we present the results of the numerical calculations. Section III A provides a discussion of the influence of background contributions. In Sec. III B the role of form factors is investigated and in Sec. III C we address the issue of identifying "missing" resonances. Our conclusions are presented in Sec. IV.

\section{REACTION DYNAMICS}

We describe the $p\left(\gamma, K^{+}\right) \Lambda$ process in terms of hadronic degrees of freedom using an effective Lagrangian approach. In this approach, every intermediate particle in the reaction dynamics is treated as an effective field with its own characteristics such as mass, photocoupling amplitudes, and strong decay widths. The effective-field theory determines the structure of the propagators and the vertices which serve as input when calculating the different Feynman graphs contributing to the reaction process. For the propagators of the spin-1/2 baryons, pseudoscalar mesons, and (axial) vector mesons the standard expressions are used. For spin-3/2 particles, the Rarita-Schwinger form for the propagator is adopted [6]. For the sake of introducing our normalization conventions for the coupling constants, the interaction Lagrangians are summarized in the Appendix. There is some ambiguity with respect to the structure of the $K \Lambda N$ vertex in the sense that one may make use of either pseudoscalar or pseudovector coupling 
(or a combination of both). For the kaon photoproduction process this issue has been studied by several authors [7-9], but neither of the two possible schemes has as yet been identified as favorable. In this work, we have chosen the pseudoscalar option. To account for the finite extension of the hadrons, it is a common procedure to introduce a phenomenological form factor at each strong vertex. These form factors depend on a cutoff mass $\Lambda$, which sets the short-range scale of the theory. It is well known that the introduction of form factors breaks the gauge invariance of the theory at the level of the Born terms, and that this can be overcome through the introduction of contact terms. Unless specified otherwise, we have adopted the gauge restoration procedure recently suggested by Davidson and Workman [10].

In an effective-field theory, the coupling constants for each of the individual resonances are not determined by the theory itself. They are treated as free parameters which are extracted by performing a global fit of the model calculations to the available data base. In a second step, these values can be compared to quark-model predictions, although the effect of final-state interactions, which are now absorbed in the effective couplings, may somehow obscure the results. To determine the vertex couplings, we compare our model calculations to the SAPHIR database [11]. It contains 90 differential and 24 total cross-section points as well as $12 \Lambda$-recoil polarization asymmetries for photon lab energies ranging from threshold $\left(\omega_{\text {lab }}=0.91 \mathrm{GeV}\right)$ up to $2 \mathrm{GeV}$. When performing a global fit to the data, the optimum set of coupling constants is the one that produces the lowest value of $\chi^{2}$. Apart from the two main coupling constants $g_{K \Lambda p}$ and $g_{K \Sigma^{0} p}$, all the extracted resonance $(R)$ parameters $G_{R}$ are a combination of photocoupling (sometimes called a magnetic transition moment) and strong hadronic coupling. A description of the various types of $G_{R}$ and their connection to the Lagrangians is given in the Appendix.

One of the striking observations when dealing with the $p\left(\gamma, K^{+}\right) \Lambda$ process in terms of hadronic degrees of freedom, is that the Born terms on their own give rise to cross sections which largely overshoot the data. Assuming SU(3) flavor symmetry [12], the coupling constants $g_{K \Lambda p}$ and $g_{K \Sigma^{0} p}$, which serve as input parameters when computing the Born contributions, are fixed by the well-known $g_{\pi N N}$ coupling. Given the substantial mass difference between strange quarks and up/down quarks, it is well known that SU(3) symmetry is broken. Assuming that $\mathrm{SU}(3)$ symmetry is broken at a $20 \%$ level, an exact relation between the coupling constants is broken and the following ranges for the $g_{K \Lambda p}$ and $g_{K \Sigma \Sigma^{0} p}$ emerge:

$$
\begin{gathered}
-4.5 \leqslant g_{K \Lambda p} / \sqrt{4 \pi} \leqslant-3.0, \\
0.9 \leqslant g_{K \Sigma^{0} p} / \sqrt{4 \pi} \leqslant 1.3 .
\end{gathered}
$$

Using values in these ranges without any further modifications, the Born terms inevitably produce far too much strength. This becomes clear in Fig. 1, where the computed total cross section is plotted in a naive model that only retains pointlike (this means before introducing hadronic form

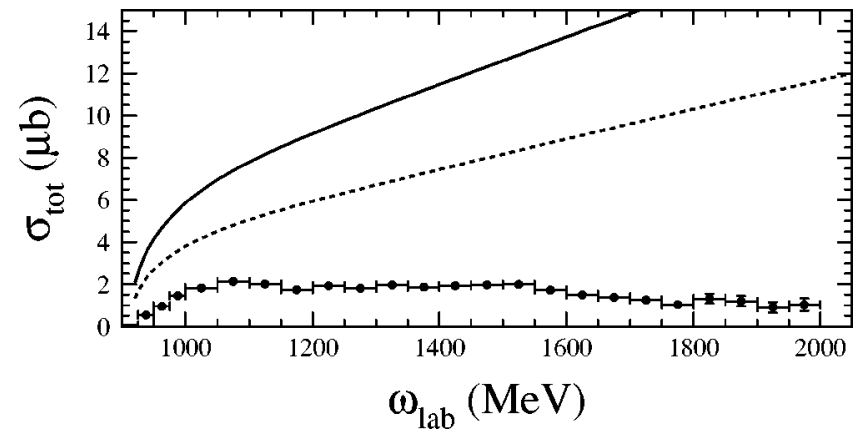

FIG. 1. The total $p\left(\gamma, K^{+}\right) \Lambda$ cross section as a function of photon lab energy as obtained when solely Born terms are included in the reaction dynamics. No hadronic form factors were introduced. For the solid line, the (unbroken) SU(3) predictions for $g_{K \Lambda p}$ and $g_{K \Sigma{ }^{0} p}$ are used while, for the dashed line, the underlimit values in Eq. (1) are taken. The data are from Ref. [11].

factors) Born terms in the reaction process. Beyond doubt, the introduction of mechanisms that reduce the Born strength is of primary concern to any model which aims at providing a realistic description of the $p\left(\gamma, K^{+}\right) \Lambda$ process. In this work we present three possible schemes that accomplish this goal. The first two schemes respect the ranges for the magnitude of the coupling constants imposed by (broken) SU(3) symmetry as written in Eq. (1), the third one does not.

(i) The introduction of hadronic form factors is well known to reduce the strength stemming from the Born terms. The smaller the cutoff mass $\Lambda$, the larger the reduction. In order to sufficiently cut the strength from the Born terms without any further modifications of the theoretical framework, the introduction of (unrealistically) small cutoff masses appears necessary $[13,14]$.

(ii) A second option for counterbalancing the strength from the Born terms is the introduction of hyperon resonances in the $u$ channel $[15,16]$. We have shown [13] that $u$ channel hyperon resonances destructively interfere with the Born terms, thereby reducing the total amount of strength to a level that appears realistic.

(iii) A third option consists of simply ignoring the ranges for the coupling constants of Eq. (1) [8]. This inevitably amounts to using coupling constants that are significantly smaller than what is expected on the basis of (broken) SU(3) symmetry.

Recent analyses $[2,14,17]$ found that three nucleon resonances dominate the $p\left(\gamma, K^{+}\right) \Lambda$ reaction dynamics: $S_{11}(1650), P_{11}(1710)$, and $P_{13}(1720)$. The occurrence of an additional "new" resonance (a $D_{13}$ state) was proposed by the George Washington group [14]. This resonance naturally explains the structure at an invariant mass of about 1900 $\mathrm{MeV}$ in the energy dependence of the total cross section data from the SAPHIR Collaboration [11]. The $D_{13}(1895)$ resonance remained unobserved in pion-induced and $(\gamma, \pi)$ reactions, but its existence was predicted by the constituent quark-model calculations of Ref. [1]. As such, it appears as an appropriate candidate for one of the "missing" resonances that have long been sought for.

For the sake of clarity, here we give a definition of the background and resonant contributions. The term resonant 
TABLE I. Schematic description of the three models used to treat the background terms. The SU(3) restrictions for the $g_{K \Lambda p}$ and $g_{K \Sigma^{0} p}$ coupling constants refer to the ranges determined in Eq. (1). The cutoff masses are those of the hadronic form factors introduced in the Born terms. Also given in the table is the underlimit for $\Lambda$ imposed in the fitting procedure and the value of $\Lambda$ corresponding with the lowest value of $\chi^{2}$ (denoted as the best value). The shown $\chi^{2}$ values are those for the best fit of the specific model to the complete SAPHIR data set.

\begin{tabular}{lccccc}
\hline \hline Model & \multirow{2}{*}{ SU(3) restrictions } & \multicolumn{2}{c}{$\Lambda$ cutoff mass $(\mathrm{GeV})$} & $Y^{*}$ in $u$ channel & \multirow{2}{*}{$\chi^{2}$} \\
& underlimit & best value & & \\
\hline A & yes & $\geqslant 0.4$ & 0.4 & $\Lambda^{*}(1800) \Lambda^{*}(1810)$ & 2.99 \\
B & yes & $\geqslant 1.5$ & 1.5 & no & 2.85 \\
$\mathrm{C}$ & no & $\geqslant 1.1$ & 1.8 & \\
\hline \hline
\end{tabular}

part refers exclusively to the $s$ channel (nucleon) resonance contributions. These are the $S_{11}(1650), \quad P_{11}(1710)$, $P_{13}(1720)$, and $D_{13}(1895)$ resonances unless specified otherwise. The Born terms, two $t$ channel contributions involving the vector meson $K^{*}(892)$ and the axial vector meson $K_{1}(1270)$, and the $u$ channel hyperon resonances, which will be introduced at some point, all contribute to what is called the background. Note that resonances in the $t$ and $u$ channels do not "resonate," since their poles are beyond the physical plane of the reaction.

\section{RESULTS AND DISCUSSION}

\section{A. Background contributions}

As detailed in Sec. II, an effective Lagrangian approach to the $p\left(\gamma, K^{+}\right) \Lambda$ process requires additional mechanisms to counterbalance the unreasonable amounts of strength arising from pointlike Born terms. We have performed model calculations using each of the three different techniques to deal with the background contributions described in Sec. II. We refer to the three different treatments as models A, B, and C, and their major features are summarized in Table I.

In model A, the "background" is restricted to the Born terms and $t$-channel diagrams involving the $K^{*}$ vector meson and $K_{1}$ axial vector meson exchange. In addition, we imposed an underlimit of $0.4 \mathrm{GeV}$ for the (freely varying) value of the cutoff mass $\Lambda$ of the hadronic form factors used in the Born diagrams during the fitting procedure. It emerges that the best fits to the data were obtained with values of $\Lambda$ that approach this imposed underlimit corresponding with an extremely soft hadronic form factor. As can be seen in Fig. 2(a), the energy dependence of the background (with $\Lambda$ $=0.4 \mathrm{GeV}$ ) is smooth and steadily rising. Concerning the contributions from the resonant terms in model $\mathrm{A}$, the strength produced by $P_{13}(1720)$ is rather small, and the structure about photon lab energies of $1.5 \mathrm{GeV}$ is clearly dominated by $D_{13}(1895)$. Despite the fair agreement with the data reached in model $\mathrm{A}$, one can raise serious doubts about the realistic character of cutoff masses as small as the kaon mass [13]. Indeed, a form factor represents a purely phenomenological description of the short-range dynamics, and sets a short-distance scale beyond which the theory is believed to fail. With cutoff masses approaching the kaon mass, the form factor will unavoidably start to play a pre- dominant role in a theoretical description of the reaction dynamics, which is a rather unsatisfactory situation for an effective theory.

In model $\mathrm{B}$, we have extended the background with hyperon resonances $\left[\Lambda^{*}(1800)\right.$ and $\left.\Lambda^{*}(1810)\right]$ in the $u$ channel. Through destructive interference, the total background strength gets reduced to acceptable levels [see Fig. 2(b)], a virtue which is now reached with realistic values of the cutoff mass of the order $1.5 \mathrm{GeV}$. The hyperon coupling constants which arise from the fits are relatively large $\left[G_{\Lambda *(1800)}=-4.38\right.$ and $\left.G_{\Lambda *(1810)}=-1.75\right]$, and can be subject to discussion. To clarify this issue, we have performed fits to the data using a model which introduces seven spin$1 / 2$ hyperon resonances in the $u$ channel. The same qualitative effect was observed, but now with smaller values for $G_{Y^{*}}$. In light of these findings we argue that the two hyperon resonances which were introduced in model B could be interpreted as effective particles which account for a larger set of hyperon resonances participating in the process. Note that $u$ channel resonances do not reach their pole, and only have a smooth energy behavior. From Fig. 2 it becomes clear that the final result for the total cross section calculated in model $\mathrm{B}$ displays a more complicated pattern than what is typically observed for model A. Whereas model A predicts that the resonances peak at their corresponding invariant masses, in model $\mathrm{B}$ a rather complex interference pattern (especially at higher photon energies) between the different resonances appears.

As a third option (model C) for controlling the magnitude of the background contributions, we have performed a set of fits to the data where we ignored the restrictions imposed by broken SU(3) symmetry. We only put limitations on the signs of $g_{K \Lambda p}$ and $g_{K \Sigma \Sigma^{0} p}$. Completely analogous to model $\mathrm{A}$, in model $\mathrm{C}$ the background consists of the Born diagrams and the two spin-1 $t$ channel contributions. An underlimit of 1.1 $\mathrm{GeV}$ was imposed for the Born term form factor cutoff mass but, during the fit, $\Lambda$ arrived at a rather "hard" value of 1.8 $\mathrm{GeV}$. Also in this model, the data can be reasonably well described. Nevertheless, the overall best fit was obtained for a value $g_{K \Lambda p}=-0.40$, which is far below the $\mathrm{SU}(3)$ prediction of -3.75 .

All three techniques to deal with the background terms eventually lead to a fair agreement of the model calculations with the available data. To illustrate this, Table I summarizes 


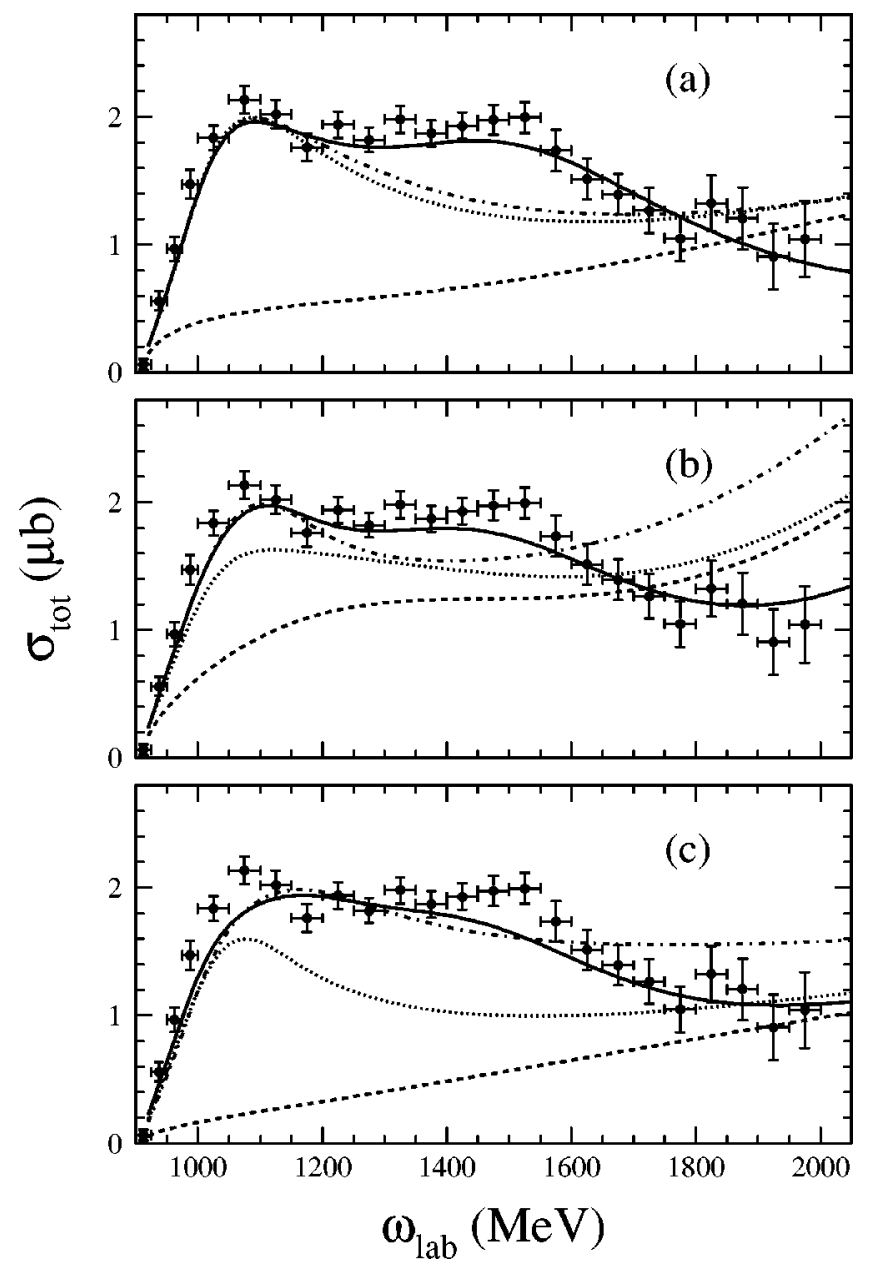

FIG. 2. The total $p\left(\gamma, K^{+}\right) \Lambda$ cross section vs the photon lab energy as obtained with three different techniques to treat the background contributions. Panels (a), (b), and (c) use models A, B, and $\mathrm{C}$, respectively. In each panel, the contribution from the background terms to the total cross sections is denoted by a dashed line. In addition to the background terms, the dotted line includes the $S_{11}(1650)$ and the $P_{11}(1710)$ nucleon resonances. The dot-dashed curve also adds the $P_{13}(1720)$ resonance. Finally, for the solid line the $D_{13}(1895)$ resonance is also included. The data are from Ref. [11]. the $\chi^{2}$ per degree of freedom obtained in the three models. Despite the fact that the $\chi^{2}$ values are comparable, Fig. 3 clearly shows that the extracted values for the $N^{*}$ coupling constants (as defined in the Appendix) differ drastically in the three models. From this observation we draw the conclusion that the model assumptions with respect to the treatment of the background terms heavily influence the extracted information about the resonances. Remarkably, it appears that the choices made with respect to modeling the background terms not only affect the magnitude of the different $N^{*}$ contributions, but also the interference pattern between the overlapping resonances (see Fig. 2).

In addition to the three frameworks to deal with the background presented here, one could think of a fourth type of model to reduce the strength stemming from the Born terms: other nucleon resonances beyond the set consisting of $S_{11}(1650), P_{11}(1710), P_{13}(1720)$, and $D_{13}(1895)$ could be introduced as likely candidates for playing a significant role in the $p\left(\gamma, K^{+}\right) \Lambda$ reaction dynamics. We have performed calculations introducing two additional spin-1/2 $N^{*}$ 's in the $s$ channel so that the number of parameters was not larger than in the models A, B, and C. In these computations, the cutoff mass $\Lambda$ was forced to adopt (realistic) values larger than 1.1 $\mathrm{GeV}$. None of the numerical calculations reached a $\chi^{2}$ better than 8 (which has to be compared to typical values of $\chi^{2}$ $\approx 2.9$ produced by the other models). From these observations, we excluded this option. In other words, the introduction of a few extra resonances in the $s$ channel cannot be invoked as a viable mechanism for cutting down the background strength.

\section{B. Hadronic form factors}

Due to the internal structure of the hadrons, the vertices cannot be treated as pointlike interactions. Therefore it is a widely adopted procedure [18] to modify each hadronic vertex with a dipole form factor of the type

$$
F_{x}(\Lambda)=\frac{\Lambda^{4}}{\Lambda^{4}+\left(x-M_{x}^{2}\right)^{2}} \quad(x \equiv s, t, u) .
$$

Herein, $\Lambda$ is the cutoff value and $x$ represents the off-shell

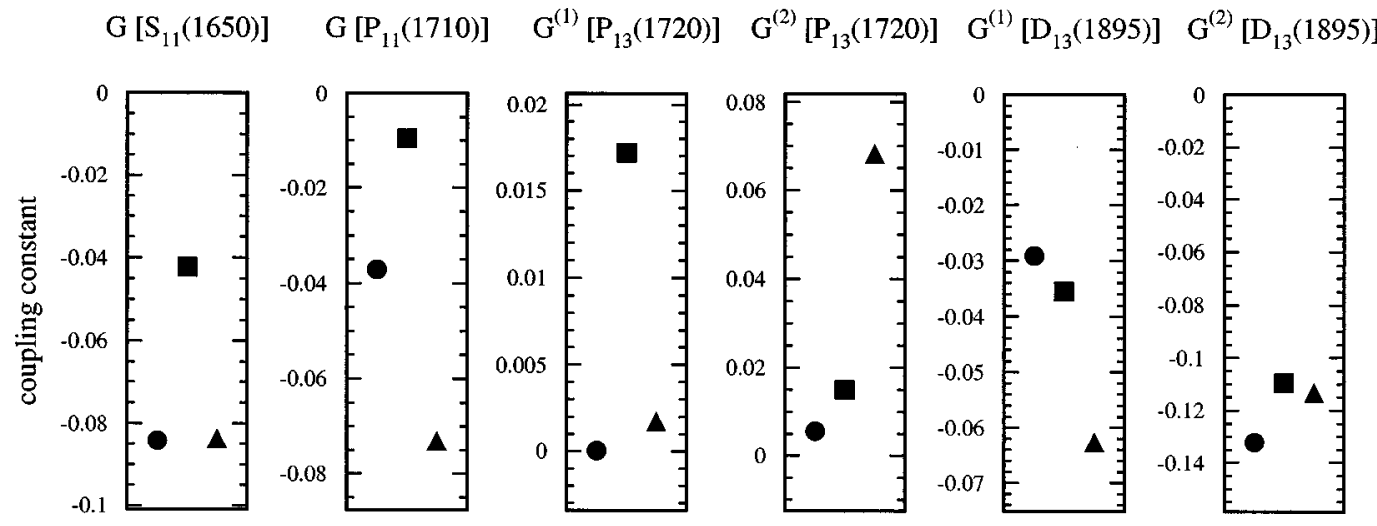

FIG. 3. The extracted coupling constants for the $S_{11}(1650), P_{11}(1710), P_{13}(1720)$, and $D_{13}(1895) s$ channel resonances using three different models $\mathrm{A}, \mathrm{B}$, and $\mathrm{C}$ for dealing with the background terms. The circles are for model $\mathrm{A}$, the squares for model $\mathrm{B}$, and the triangles for model C. 
momentum at the vertex. We note that there is some arbitrariness in the functional form of the form factor. A major implication of introducing hadronic form factors is that gauge invariance is broken at the level of the Born terms. We remark that the (axial) vector meson and resonance exchange terms, which are characterized by the electromagnetic interaction Lagrangians of types (A7), (A8), (A12) and (A16), are gauge invariant by construction. As suggested by Haberzettl et al. [19], the gauge invariance of the Born terms can be restored by adding an additional contact term, which introduces a new form factor $\hat{F}$. This contact term is determined in such a manner that the gauge violating terms are exactly canceled. For the $p\left(\gamma, K^{+}\right) \Lambda$ case it reads

$$
\begin{aligned}
\varepsilon_{\mu} \mathcal{M}_{\text {contact }}^{\mu}= & e g_{K \Lambda p} \bar{u}_{Y} \gamma_{5} \varepsilon_{\mu}\left[\frac{2 p^{\mu}}{s-M_{p}^{2}}\left(\hat{F}-F_{s}\right)\right. \\
& \left.+\frac{2 p_{K}^{\mu}}{t-M_{K}^{2}}\left(\hat{F}-F_{t}\right)\right] u_{p},
\end{aligned}
$$

where $p^{\mu}\left(p_{K}^{\mu}\right)$ is the proton (kaon) 4-momentum, and $F_{s}(\Lambda)$ and $F_{t}$ are given in Eq. (2).

Recently, Davidson and Workman [10] criticized the functional form of $\hat{F}$ proposed by Haberzettl et al. (hereafter denoted by $\hat{F}_{H}$ ). They showed that with $\hat{F}_{H}$, the contact term of Eq. (3) is not free of poles, and consequently flawed. At the same time, the authors suggested an alternate recipe for the form factor (hereafter, denoted as $\hat{F}_{D W}$ ). For a detailed discussion we refer to the original papers. Here we just report the global form of the two different recipes:

$$
\begin{aligned}
& \hat{F}_{H}=a_{s} F_{s}(\Lambda)+a_{t} F_{t}(\Lambda)+a_{u} F_{u}(\Lambda), \\
& \hat{F}_{D W}=F_{s}(\Lambda)+F_{t}(\Lambda)-F_{s}(\Lambda) F_{t}(\Lambda),
\end{aligned}
$$

where the $a_{x}$ coefficients in Eq. (4) have to satisfy the relation $a_{s}+a_{t}+a_{u}=1$.

We have performed numerical calculations using both the $\hat{F}_{H}$ and $\hat{F}_{D W}$ functional forms in the contact term. For those numerical calculations using $\hat{F}_{H}$, we have put $a_{u}=0$. This choice is motivated by the observation that in the $p\left(\gamma, K^{+}\right) \Lambda$ process, the gauge violating terms only occur in the $s$ and $t$ channels. As a result, calculations using the $\hat{F}_{H}$ form have two remaining free parameters ( $\Lambda$ and $a_{s}$ ), stemming from the form factors and the gauge restoring procedure. In practice, we found that the best fits were obtained for $a_{s} \approx 1$ and accordingly $\hat{F}_{H} \approx F_{S}(\Lambda)$. Figure 4 compares the values of $\hat{F}_{H}$ and $\hat{F}_{D W}$ at various photon energies $\omega_{\text {lab }}$ and kaon centerof-mass angles $\theta$. The left panels show the form factors for a cutoff mass $\Lambda=0.8 \mathrm{GeV}$, and the right panels use $\Lambda=1.8$ $\mathrm{GeV}$. They are representative of rather "soft" $(\Lambda=0.8 \mathrm{GeV})$ and "hard" $(\Lambda=1.8 \mathrm{GeV})$ options for the form factors. It becomes obvious from Fig. 4 that the magnitude of the form factor $\hat{F}$ depends heavily on the adopted recipe.

In order to assess the sensitivity of the results to differences in the functional form of $\hat{F}$, we computed $p\left(\gamma, K^{+}\right) \Lambda$
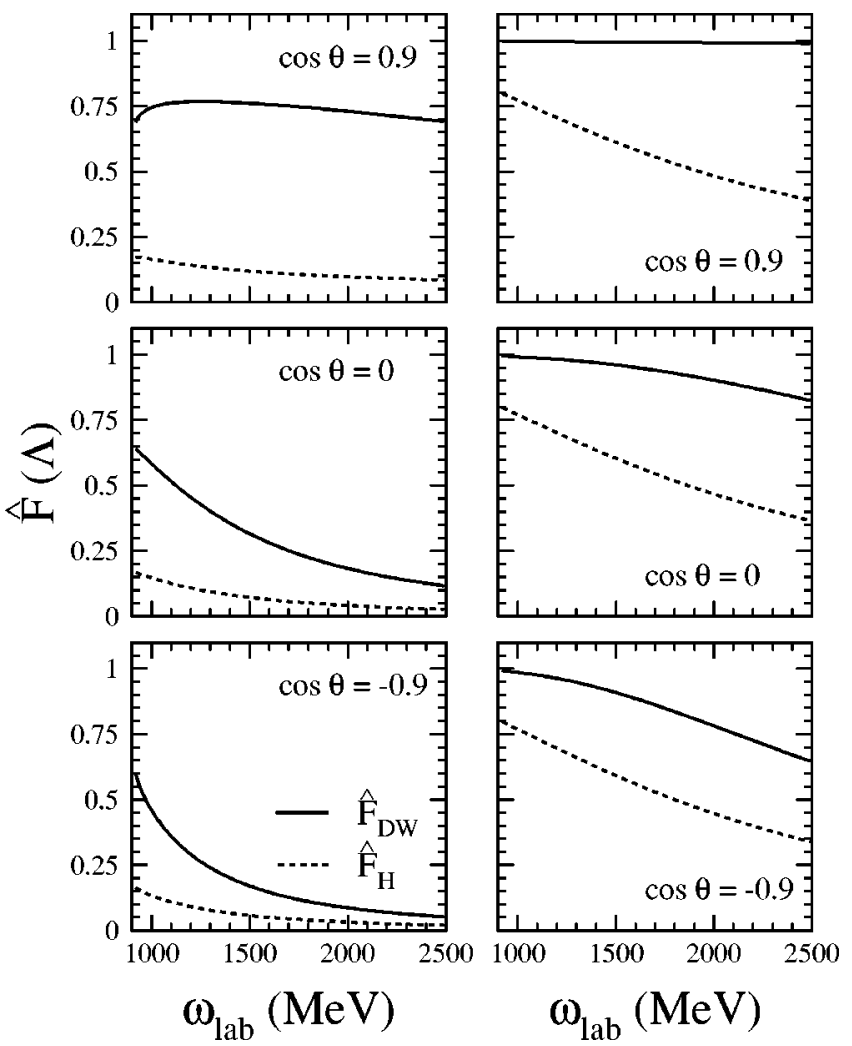

FIG. 4. The energy dependence of the hadronic form factor $\hat{F}$ for different kaon center-of-mass angles $\theta$. The left panels use $\Lambda$ $=0.8 \mathrm{GeV}$ and the right panels $\Lambda=1.8 \mathrm{GeV}$. The dashed line is the Haberzettl form (with $a_{s} \sim 0.9$ ). The solid lines represent the form proposed by Davidson and Workman.

observables using the two forms of $\hat{F}$. The results for the extracted $N^{*}$ coupling constants are given in Fig. 5 for models $\mathrm{A}$ and $\mathrm{B}$. In model A, where by construction a large role in the reaction dynamics is attributed to form factors, the effect is enormous. In model B, where hyperon resonances are introduced to counterbalance the strength from the Born terms and hadronic form factors are not so dominant, the extracted coupling constants are generally more stable against variations in the functional dependence of $\hat{F}$, although here sizable variations are also observed. A similar trend is seen, for example, in the photon beam asymmetry (see Fig. 6). Whereas for model A different choices of $\hat{F}$ even switch the sign of the predicted asymmetry, in model B the situation looks reasonably stable. Only at the highest photon energies considered here, the predicted asymmetry in model B becomes sensitive to the adopted recipe for the form factor in the contact term.

These conclusions are compatible with earlier observations concerning the difference between the form factor prescriptions of Ohta and Haberzettl et al. Ohta originally suggested [20] setting the form factor $\hat{F}$ in the contact term equal to 1 . As becomes clear from Fig. 4 , the recipe for $\hat{F}$ suggested by Davidson and Workman gives rise to values between those produced by the Haberzettl et al. and Ohta forms. In several works $[2,9,19]$, it was stressed that $p\left(\gamma, K^{+}\right) \Lambda$ calculations with Haberzettl et al. or Ohta's 


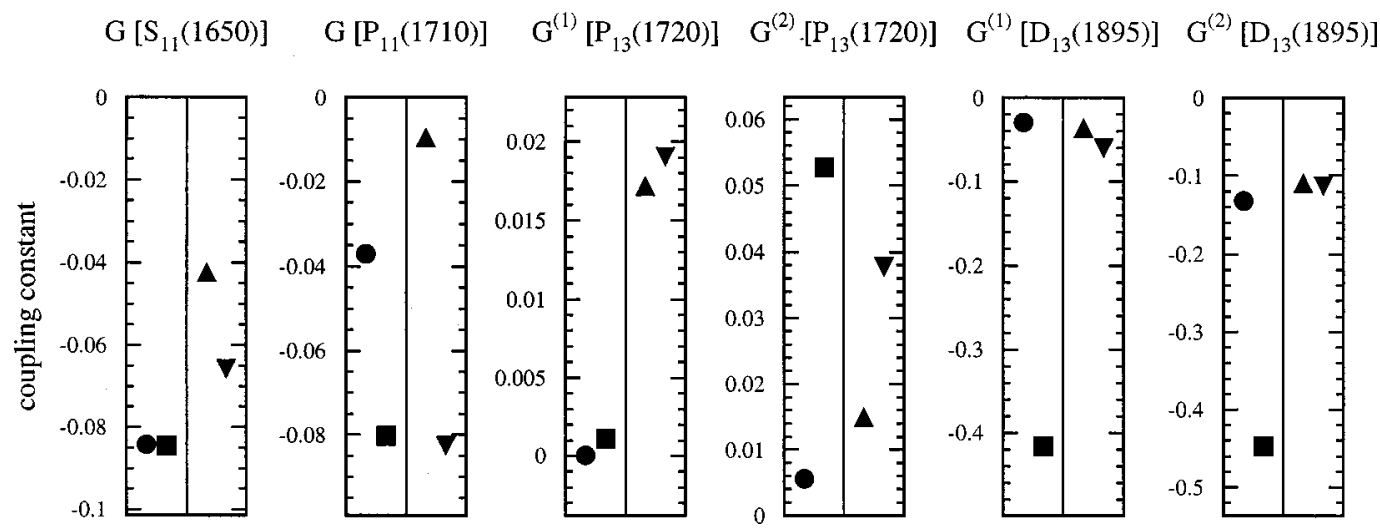

FIG. 5. The sensitivity of the extracted $N^{*}$ coupling constants to the adopted form for the hadronic form factor $\hat{F}$. The circles are obtained from calculations using the $\hat{F}_{D W}$ form, and the squares with $\hat{F}_{H}$. They both correspond to a calculation which uses model A to treat the background contributions. Analogously, the triangles $\boldsymbol{\Delta}(\boldsymbol{\nabla})$ are for the $\hat{F}_{D W}\left(\hat{F}_{H}\right)$ form in model B.

recipe for $\hat{F}$ can lead to very different results. So, in fact, it comes as no real surprise that effects are substantial.

In Ref. [21], Davidson and Workman studied the effect of hadronic form factors on a multipole analysis of charged pion production. They concluded that extracted multipoles, for example those listed in Ref. [22], are not heavily affected by the form factors. Our calculations indicate that, for kaon photoproduction, where the effect of the background terms is larger than in the pion case, great care must be taken when introducing hadronic form factors and the corresponding gauge-restoring contact terms.

We conclude this section with a more general remark. In principle, a correction to a hadronic form factor is not supposed to have a large impact on the reaction dynamics. At best, hadronic form factors are a purely phenomenological tool to smooth the (unknown) high-energy behavior of the effective-field theory. If, for some reason, the influence turns out to be large, it is obvious that one runs into a rather unsatisfactory situation. In that respect, the introduction of soft hadronic form factors (model A) in modeling the kaon
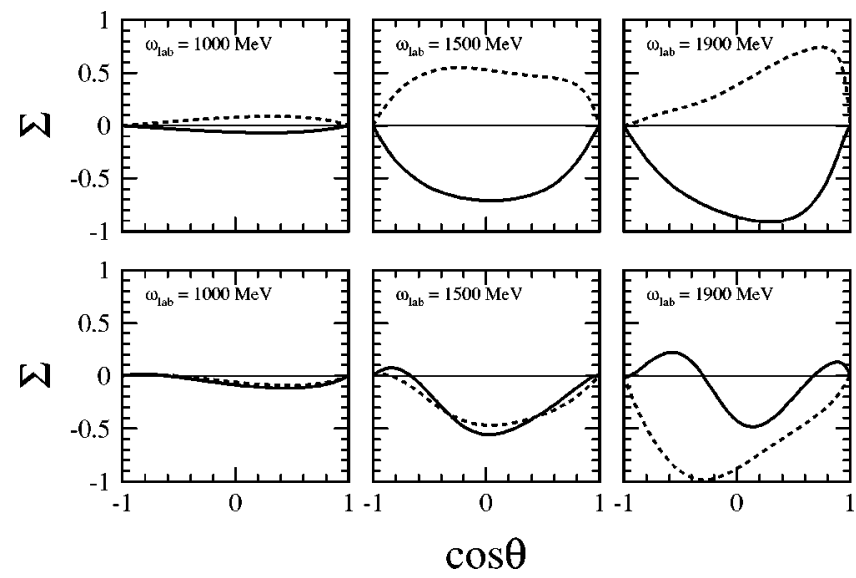

FIG. 6. The angular distribution of the beam polarization asymmetry for $p\left(\vec{\gamma}, K^{+}\right) \Lambda$ at three photon lab energies. The upper (lower) panels are results with model A (model B) for treating the background diagrams. Solid and dashed lines use the hadronic form factors $\hat{F}_{D W}$ and $\hat{F}_{H}$, respectively. photoproduction process, appears to lead to an unacceptable level of (unphysical) model dependency in the extracted information from fits to $p\left(\gamma, K^{+}\right) \Lambda$ data.

\section{Missing resonances}

The SAPHIR data [11], released in 1998, made it clear that the total $p\left(\gamma, K^{+}\right) \Lambda$ cross section is not characterized by a smooth energy dependence above the threshold peak. The data displayed a structure about photon lab energies of 1.5 $\mathrm{GeV}$. Mart and Bennhold [14] interpreted this structure as evidence of an additional resonance, and they identified it as a $D_{13}$ state with a mass of $1895 \mathrm{MeV}$. This $N^{*}$ state remained unobserved in pion-induced and pion photoproduction processes, but its existence and appreciable decay in the $K^{+} \Lambda$ channel was inferred from the constituent quark calculations of Capstick and Roberts [1]. As such, $D_{13}(1895)$ appeared as a good candidate for a "missing" resonance. Our calculations, displayed in Fig. 7, essentially confirm the observations made in Ref. [14] and reveal that the structure at $\omega_{l a b} \sim 1.5 \mathrm{GeV}$ can be reasonably accounted for after including a $D_{13}$ resonance in the $s$ channel in the model calculations. Apart from a $D_{13}$ state, the quark-model calculations of Ref. [1] predict other $N^{*}$ 's with decay in the strange channels in the mass range about $1.9 \mathrm{GeV}$. Other candidates are $S_{11}$ (1945), $P_{11}(1975)$, and $P_{13}(1950)$. We have performed calculations adding a "missing" $P_{13}$ resonance to the basic

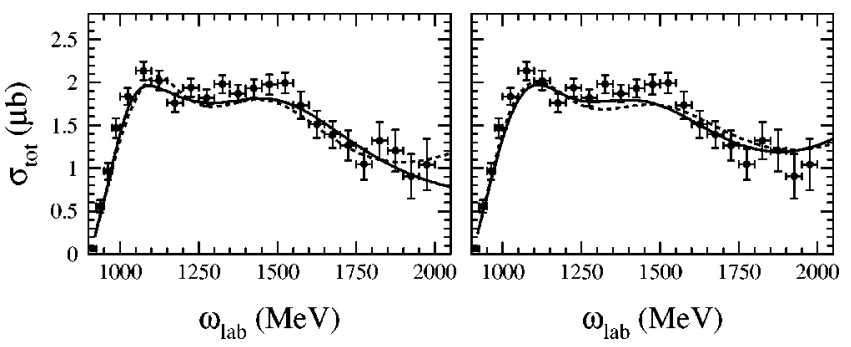

FIG. 7. Model calculations for the total $p\left(\gamma, K^{+}\right) \Lambda$ cross section. The solid curves include the "missing" $D_{13}$, and the dashed lines include a $P_{13}$ resonance. The left (right) panel uses model A (model B) to describe the background contributions. 
set of $S_{11}(1650), P_{11}(1710)$, and $P_{13}(1720)$. The results of these model calculations are also contained in Fig. 7. It is clear that the procedure of introducing either an extra $D_{13}$ or $P_{13}$ resonance does equally well in reproducing the resonant structure in the energy dependence of the total $p\left(\gamma, K^{+}\right) \Lambda$ cross section, independent of the adopted model to handle the background terms. Similar observations were already made in Ref. [14], and there is common agreement about the fact that the reproduction of a visual "bump" in the total cross section should not be interpreted as rock-solid evidence for the occurrence of a missing resonance. Nevertheless, in Ref. [14], $D_{13}$ was considered to be the preferred candidate on the basis of the agreement between the extracted coupling constants in the fits and the values predicted by the quark model. In the light of discussions of the model dependencies in Secs. III A and III B, great care must be exercised in drawing conclusions on the basis of the values of the extracted coupling constants. Furthermore, we stress that the calculations of Mart and Bennhold used Haberzettl et al.'s recipe for the form factor $\hat{F}$, and employed a relatively soft cutoff mass $(\Lambda=0.8 \mathrm{GeV})$ for the Born terms. In that respect, their model comes close to what we referred to as model A.

\section{CONCLUSION}

In summary, we have investigated the kaon photoproduction reaction for photon energies up to $2 \mathrm{GeV}$ in a fieldtheoretic approach. From our investigations it becomes clear that the treatment of background processes in the $p\left(\gamma, K^{+}\right) \Lambda$ reaction is not free from ambiguities. At the same time, the background terms influence the values of the extracted resonance parameters dramatically, which makes the extraction of model-independent information far from evident. We have also investigated how sensitive the predictions are to the adopted recipes for the phenomenological hadronic form factor $\hat{F}$ appearing in the contact terms, which are meant to restore gauge invariance. Former investigations mainly used Ohta's or Haberzettl et al.'s forms for the form factor $\hat{F}$. Davidson and Workman pointed out that both of these recipes are theoretically unacceptable, and provided an alternate prescription. We have made a systematic study of the consequences of these corrections for the computed $p\left(\gamma, K^{+}\right) \Lambda$ observables. In the energy range under investigation, the corrections are rather large, and the effect on the extracted coupling constants is substantial. Moreover, it is clear that even an extensive and accurate data set, such as the one produced by the SAPHIR Collaboration, does not allow one to precisely determine the various contributions in the underlying $p\left(\gamma, K^{+}\right) \Lambda$ reaction dynamics. The measured cross sections and recoil polarization asymmetries do not suffice to nail down the complicated interference pattern between the various resonances. After all, this is not so surprising. It is well known that a complete meson photoproduction experiment needs at least seven observables to constrain the reaction amplitudes at a fixed photon energy [23]. As we find that the treatment of background contributions and hadronic form factors heavily influences the extracted values for the coupling constants, we deem it premature to identify the quan- tum numbers of a "missing" resonance on the basis of the existing data set. A measurement of polarization observables will be essential to further constrain the major reaction mechanisms.

As a final remark, we mention that one can raise some reservations about the applicability of theoretical models that handle resonances as purely individual particles. From a historical point of view, this technique was appropriate for pion reactions in the delta region where the process is dominated by one resonance. In the kaon photoproduction channels, a multitude of interfering resonances contribute and maybe one has to develop more advanced techniques to disentangle these resonances and their combined action.

\section{ACKNOWLEDGMENT}

This work was supported by the Fund for Scientific Research-Flanders under Contract No. 4.0061.99.

\section{APPENDIX: INTERACTION LAGRANGIANS}

The interaction Lagrangians used in meson production calculations are given in many works. For the sake of defining our notation and normalization conventions, we summarize the ones which are relevant for the $p\left(\gamma, K^{+}\right) \Lambda$ process.

\section{Born terms}

The electromagnetic interaction Lagrangians for the Born terms are given by

$$
\begin{gathered}
\mathcal{L}_{\gamma p p}=-e \bar{N} \gamma_{\mu} N A^{\mu}+\frac{e \kappa_{p}}{4 M_{p}} \bar{N} \sigma_{\mu \nu} N F^{\mu \nu}, \\
\mathcal{L}_{\gamma \Lambda \Lambda}=\frac{e \kappa_{\Lambda}}{4 M_{p}} \bar{\Lambda} \sigma_{\mu \nu} \Lambda F^{\mu \nu} \\
\mathcal{L}_{\gamma \Lambda \Sigma^{0}}=\frac{e \kappa_{\Sigma^{0} \Lambda}}{4 M_{p}} \Sigma^{0} \sigma_{\mu \nu} \Lambda F^{\mu \nu}+\text { H.c. } \\
\mathcal{L}_{\gamma K K}=-i e\left(K^{\dagger} \partial_{\mu} K-K \partial_{\mu} K^{\dagger}\right) A^{\mu} .
\end{gathered}
$$

The antisymmetric tensor for the photon field is defined as $F^{\mu \nu}=\partial^{\nu} A^{\mu}-\partial^{\mu} A^{\nu}$. For the anomalous magnetic moments we have used the values [17] $\kappa_{p}=1.793$ and $\kappa_{\Lambda}=-0.613$. For the sign of the magnetic transition moment $\kappa_{\Sigma}{ }^{0} \Lambda$, which is experimentally not accessible, we have used the de Swart convention [12] which yields $\kappa_{\Sigma}{ }^{0} \Lambda=+1.61$. For the hadronic $K \Lambda p$ interaction, a pseudoscalar (PS) or pseudovector (PV) option is viable:

$$
\begin{gathered}
\mathcal{L}_{K \Lambda p}^{P S}=-i g_{K \Lambda p} K^{\dagger} \bar{\Lambda} \gamma_{5} N+\text { H.c. } \\
\mathcal{L}_{K \Lambda p}^{P V}=\frac{f_{K \Lambda p}}{M_{K}} \partial^{\mu} K^{\dagger} \bar{\Lambda} \gamma_{\mu} \gamma_{5} N+\text { H.c. }
\end{gathered}
$$

All results in this work are obtained with the PS variant.

\section{Spin-1 meson exchange}

The electromagnetic coupling to a vector meson $(V)$ is described by 


$$
\mathcal{L}_{\gamma K V}=\frac{e \kappa_{K V}}{4 M} \epsilon^{\mu \nu \lambda \sigma} F_{\mu \nu} V_{\lambda \sigma} K
$$

where the vector meson tensor is given by $V^{\mu \nu}=\partial^{\nu} V^{\mu}$ $-\partial^{\mu} V^{\nu}$, and $V^{\mu}$ is the vector field. The photon coupling to an axial vector meson $\left(V_{a}\right)$ reads

$$
\mathcal{L}_{\gamma K V_{a}}=i \frac{e \kappa_{K V_{a}}}{M}\left(\partial_{\mu} A_{\nu} \partial^{\mu} V_{a}^{\nu}-\partial_{\mu} A_{\nu} \partial^{\nu} V_{a}^{\mu}\right) K
$$

where $V_{a}^{\mu}$ is the axial vector field. The mass scale $M$ for the transition moment is arbitrarily chosen as $1.0 \mathrm{GeV}$. The complete antisymmetric tensor is defined as $\epsilon^{0123}=1$. Note that this convention produces a sign difference to the covariant definition. The hadronic vertex has a vector $(v)$ part and a tensor $(t)$ part,

$$
\begin{aligned}
\mathcal{L}_{V \Lambda p}= & -g_{V \Lambda p}^{v} \bar{\Lambda} \Gamma_{\mu} N V^{\mu}+\frac{g_{V \Lambda p}^{t}}{2\left(M_{\Lambda}+M_{p}\right)} \bar{\Lambda} \sigma_{\mu \nu} V^{\mu \nu} \Gamma N \\
& + \text { H.c. }
\end{aligned}
$$

where $V$ is now a shorthand notation for both a vector and an axial vector meson. Furthermore, $\Gamma=1\left(\gamma^{5}\right)$ and $\Gamma^{\mu}=\gamma^{\mu}$ $\left(\gamma^{\mu} \gamma^{5}\right)$ for vector (axial vector) meson resonances. The information about coupling constants which can be extracted from fits to the data, reads

$$
\begin{gathered}
G_{V}^{v}=\frac{e g_{V \Lambda p}^{v}}{4 \pi} \kappa_{K V}, \\
G_{V}^{t}=\frac{e g_{V \Lambda p}^{t}}{4 \pi} \kappa_{K V},
\end{gathered}
$$

with $V$ a vector or axial vector meson.

\section{Spin-1/2 resonance exchange}

For spin-1/2 resonances, the electromagnetic interaction reads

$$
\mathcal{L}_{\gamma B R}=\frac{e \kappa_{B R}}{4 M_{p}} \bar{R} \Gamma_{\mu \nu} B+\text { H.c. }
$$

where the hadronic vertices are described by a pseudoscalar part or a pseudovector part:

$$
\begin{gathered}
\mathcal{L}_{K B R}^{P S}=-i g_{K B R} K^{\dagger} \bar{B} \Gamma R+\text { H.c. } \\
\mathcal{L}_{K B R}^{P V}=\frac{f_{K B R}}{M_{K}}\left(\partial^{\mu} K^{\dagger}\right) \bar{B} \Gamma_{\mu} R+\text { H.c. }
\end{gathered}
$$

Herein, $\Gamma^{\mu \nu}=\gamma^{5} \sigma^{\mu \nu}\left(\sigma^{\mu \nu}\right)$ for odd (even) parity resonances. $\Gamma$ and $\Gamma^{\mu}$ are defined as before. Further, $B$ is the baryon field (a $N$ or $\Lambda$ depending on the corresponding vertex) and $R$ is the spin-1/2 baryon resonance field (a $N^{*}$ or $Y^{*}$ ). In this work we have only used the PS scheme. For spin-1/2 resonance exchange, the information regarding the extracted coupling constant takes on the form

$$
G_{R}=\frac{g_{K B R}}{\sqrt{4 \pi}} \kappa_{B R}
$$

\section{Spin-3/2 resonance exchange}

For spin-3/2 resonances, there are two terms in the Lagrangian describing the electromagnetic interaction:

$$
\begin{aligned}
\mathcal{L}_{\gamma B R}= & i \frac{e \kappa_{B R}^{(1)}}{2 M_{p}} \bar{R}^{\mu} \theta_{\mu \nu}(Y) \Gamma_{\lambda} B F^{\lambda \nu} \\
& -\frac{e \kappa_{B R}^{(2)}}{4 M_{p}^{2}} \bar{R}^{\mu} \theta_{\mu \nu}(X) \Gamma\left(\partial_{\lambda} B\right) F^{\nu \lambda}+\text { H.c. }
\end{aligned}
$$

The hadronic vertex is given by

$$
\mathcal{L}_{K B R}=\frac{f_{K B R}}{M_{K}} \bar{R}^{\mu} \theta_{\mu \nu}(Z) \Gamma^{\prime} B\left(\partial^{\nu} K\right)+\text { H.c. }
$$

Here $\Gamma$ and $\Gamma^{\mu}$ are defined as above and $\Gamma^{\prime}=\gamma^{5}(1)$ for odd (even) parity resonances. The function $\theta_{\mu \nu}(V)$ reflects the invariance of the free Lagrangian of a spin-3/2 field under a point transformation [6], and is given by

$$
\theta_{\mu \nu}(V)=g_{\mu \nu}-\left(V+\frac{1}{2}\right) \gamma_{\mu} \gamma_{\nu}
$$

The parameters $V=X, Y, Z$ are the so-called off-shell parameters. For spin-3/2 resonance exchange, the fits of the model calculations to the data give access to the following combination of coupling constants:

$$
\begin{aligned}
G_{R}^{(1)} & =\frac{e f_{K B R}}{4 \pi} \kappa_{B R}^{(1)}, \\
G_{R}^{(2)} & =\frac{e f_{K B R}}{4 \pi} \kappa_{B R}^{(2)} .
\end{aligned}
$$

[1] S. Capstick and W. Roberts, Phys. Rev. D 58, 074011 (1998).

[2] T. Feuster and U. Mosel, Phys. Rev. C 59, 460 (1999).

[3] T. Vrana, S. Dytman, and T.-S. H. Lee, Phys. Rep. 328, 181 (1999).
[4] D. Manley and E. Saleski, Phys. Rev. D 45, 4002 (1992).

[5] W.-T. Chiang, F. Tabakin, and T.-S. Lee, Phys. Lett. B 517, 101 (2001).

[6] M. Benmerrouche, R. Davidson, and N. Mukhopadhyay, Phys. Rev. C 39, 2339 (1989). 
[7] C. Bennhold and L. Wright, Phys. Rev. C 36, 438 (1987).

[8] S. Hsiao, D. Lu, and S. Yang, Phys. Rev. C 61, 068201 (2000).

[9] B. Han, M. Cheoun, K. Kim, and I.-T. Cheon, Nucl. Phys. A691, 713 (2001).

[10] R. Davidson and R. Workman, Phys. Rev. C 63, 025210 (2001).

[11] M. Q. Tran et al., Phys. Lett. B 445, 20 (1998); http:// lisa12.physik.uni-bonn.de/saphir/klks.txt

[12] J. de Swart, Rev. Mod. Phys. 35, 916 (1963).

[13] S. Janssen, J. Ryckebusch, W. Van Nespen, D. Debruyne, and T. Van Cauteren, Eur. Phys. J. A 11, 181 (2001).

[14] T. Mart and C. Bennhold, Phys. Rev. C 61, 012201(R) (2000).

[15] R. Adelseck and B. Saghai, Phys. Rev. C 42, 108 (1990).
[16] J. David, C. Fayard, G. Lamot, and B. Saghai, Phys. Rev. C 53, 2613 (1996).

[17] Particle Data Group, D. E. Groom et al., Eur. Phys. J. C 15, 1 (2000).

[18] B. Pearce and B. Jennings, Nucl. Phys. A528, 655 (1991).

[19] H. Haberzettl, C. Bennhold, T. Mart, and T. Feuster, Phys. Rev. C 58, R40 (1998).

[20] K. Ohta, Phys. Rev. C 40, 1335 (1989).

[21] R. Davidson and R. Workman, Phys. Rev. C 63, 058201 (2001).

[22] R. Arndt, R. Workman, Z. Li, and L. Roper, Phys. Rev. C 42 , 1853 (1990).

[23] I. Baker, A. Donnachie, and J. Storrow, Nucl. Phys. B95, 347 (1975). 